\title{
A Gravity Dual of Chiral Symmetry Breaking
}

\author{
J. Babington ${ }^{1}$, J. Erdmenger ${ }^{1}$, N. Evans ${ }^{2}$, Z. Guralnik ${ }^{1}$, I. Kirsch ${ }^{1}$ * \\ ${ }^{1}$ Institut für Physik, Humboldt-Universität zu Berlin \\ Newtonstraße 15, D-12489 Berlin, Germany \\ ${ }^{2}$ Department of Physics, Southampton University \\ Southampton S017 1BJ, United Kingdom
}

\begin{abstract}
We establish a holographic description of chiral symmetry breaking for a particular confining large $N$ non-supersymmetric gauge theory with matter in the fundamental representation. The gravity dual of this theory is obtained by introducing a D7-brane probe into a deformed Anti-de Sitter background found by Constable and Myers. We numerically compute the $\bar{\psi} \psi$ condensate and meson spectrum by solving the equation of motion of the D7-brane probe in this background. We find a symmetry breaking condensate as well as the associated, pion-like, Goldstone boson in the limit of small quark mass. The existence of the condensate ensures that the D7-brane never reaches the naked singularity at the origin of the deformed AdS space.
\end{abstract}

\section{Introduction}

Generalizations of AdS/CFT duality [1] in which supersymmetry and conformal symmetry are broken have led to promising new ideas for studying strong coupling phenomena in large $N$ gauge theories. In particular, it is hoped that methods based on gauge-gravity duality will eventually be applicable to QCD. Notable progress towards this goal has been achieved by considering asymptotically AdS geometries which correspond to renormalization group (RG) flows from a super-conformal gauge theory in the ultraviolet to a QCD-like theory in the infrared. A number of non-supersymmetric ten-dimensional geometries of this form have been found 2, 3, 4, 5, and been shown to describe confining gauge dynamics. There have been many interesting calculations of the glueball spectrum in three- and four-dimensional QCD-like theories in such backgrounds (see for instance [6]). These spectra are obtained by solving the classical supergravity equations of motion. While these geometries are actually strong-coupling descriptions of gauge theories with extra massive degrees of freedom in addition to those of pure QCD, the glueball spectra still compare favorably with lattice data [7.

To take a step closer to finding a holographic dual of QCD, it is necessary to include particles in the fundamental representation of the gauge group, i.e. quarks. The addition of fundamental degrees of freedom to the dual gauge theory is achieved by embedding an appropriate D-brane probe in the gravity background. Holography in the presence of a probe brane was studied in detail in relation to defect conformal field theories [8, 9, 10,

*corresponding author E-mail: ik@physik.hu-berlin.de 
To obtain a holographic dual of a four-dimensional gauge theory with fundamental matter, Karch and Katz [11] considered a configuration in which a D7-brane in $A d S_{5} \times S^{5}$ fills $A d S_{5}$ and wraps an $S^{3}$ inside $S^{5}$. This configuration is the near-horizon limit of $N$ D3-branes sharing three spatial directions with a single D7-brane. The open string degrees of freedom are those of the $\mathcal{N}=4$ super Yang-Mills theory, coupled to an $\mathcal{N}=2$ hypermultiplet in the fundamental representation of $S U(N)$. The latter arises from strings stretched between the D7- and D3-branes. When the D7 and D3's are separated, the fundamental matter becomes massive and the dual description of the probe D7-brane has an induced metric that is only asymptotically $A d S_{5} \times S^{3}$. In this case there is a discrete spectrum of mesons. This spectrum has been computed (exactly!) at large 't Hooft coupling [12] using an approach analogous to the glueball calculations in deformed AdS backgrounds. The novel feature here is that the "quark" bound states are described by the scalar fields in the Dirac-Born-Infeld (DBI) action of the D7-brane probe. Neglecting the back-reaction of the probe on the geometry corresponds to the quenched approximation of the gauge theory. - Further related work on embedding a D7 probe into the KlebanovStrassler background may be found in 13 .

In this talk we consider one of the most important features of QCD dynamics, which is chiral symmetry breaking by a $\bar{\psi} \psi$ condensate. This occurs only in non-supersymmetric theories. Therefore we consider as in [14] the embedding of a D7-brane probe into the non-supersymmetric background of Constable and Myers [5], which exhibits confinement. In this holographic setting we do indeed find chiral symmetry breaking.

The Constable-Myers background is asymptotically $A d S_{5} \times S^{5}$, but has a non-constant dilaton and $S^{5}$ radius. In the dual gauge theory this corresponds to the addition of an operator of dimension 4 with zero R-charge. This generates an $\mathrm{RG}$ flow from $\mathcal{N}=4$ super Yang-Mills to a non-supersymmetric gauge theory. The gauge theory does not quite have the field content of $\mathrm{QCD}$, since the adjoint fermions and scalars of the ultraviolet $\mathcal{N}=4$ theory do not decouple. Nevertheless, in a certain parameter range, the geometry leads to an area law for the Wilson loop, corresponding to confinement in the dual gauge theory. Solving the classical gravity equations also gives a discrete spectrum of glueballs with a mass gap.

We obtain numerical solutions for the D7-brane equations of motion in the ConstableMyers background with UV asymptotic behavior determined by a quark mass and a chiral condensate. The quark mass $m$ and the quark condensate expectation value $c$ are given by the UV asymptotic behavior of the solutions to the Dirac-Born-Infeld equations of motion in the standard holographic way. ${ }^{1}$ Using a numerical 'shooting' technique, we compute the condensate $c$ as a function of the quark mass $m$ by imposing a regularity constraint. We find that there are regular solutions with non-vanishing chiral condensate.

A significant feature of the Constable-Myers geometry is that it has a naked singularity in the far infrared, whose interpretation is a delicate issue, for instance in the light of the analysis of [15]. Remarkably, our results are not sensitive to the singular behavior of the metric in the IR. For all the solutions of the D7 equation of motion satisfying the regularity constraint, the D7 "ends" before reaching the curvature singularity. Of course the D7-brane does not really end, however the $S^{3}$ about which it is wrapped contracts to zero size, in a manner similar to a scenario discussed in [11]. The screening of the singularity is related to the existence of the condensate, which persists even in the limit $m \rightarrow 0$. This corresponds to spontaneous breaking of a $U(1)$ chiral symmetry of the

\footnotetext{
${ }^{1}$ In the $\mathcal{N}=2$ supersymmetric scenario of 11 with a D7-brane probe in standard AdS space, there cannot be any regular solution which has $c \neq 0$; the supersymmetric theory does not allow a quark condensate. We confirmed this by an explicit calculation.
} 
dual gauge theory. In the probe geometry this $U(1)$ is the rotation symmetry in the two directions orthogonal to the D7-brane worldvolume. We emphasize that this $U(1)$ chiral symmetry is non-anomalous in the large $N$ limit [16], which is also the limit in which the classical supergravity approximation is valid.

We also compute the meson spectrum by studying classical fluctuations about the D7-embedding. In agreement with spontaneous chiral symmetry breaking, the meson spectrum contains a massless mode in the limit of zero quark mass.

For subsequent developments concerning gauge/gravity duality with flavor see Refs. 17, 18, 19, 20, 21. This note is based on a talk given by I. Kirsch at the 36th Symposium Ahrenshoop: Recent Developments in String/M-Theory and Field Theory, August 2003.

\section{$2 \quad$ D7 probe brane in Constable-Myers background}

We choose an appropriate coordinate system for the background of [5] such that in Einstein frame, the geometry is given by

$$
d s^{2}=H^{-1 / 2}\left(\frac{w^{4}+b^{4}}{w^{4}-b^{4}}\right)^{\delta / 4} \sum_{j=0}^{3} d x_{j}^{2}+H^{1 / 2}\left(\frac{w^{4}+b^{4}}{w^{4}-b^{4}}\right)^{(2-\delta) / 4} \frac{w^{4}-b^{4}}{w^{4}} \sum_{i=1}^{6} d w_{i}^{2},
$$

where $b$ is the parameter of the geometry that determines the size of the deformation $\left(\delta=L^{4} /\left(2 b^{4}\right)\right.$ with $L$ the AdS radius) and

$$
H=\left(\frac{w^{4}+b^{4}}{w^{4}-b^{4}}\right)^{\delta}-1, \quad w^{2}=\sum_{i=1}^{6} w_{i}^{2} .
$$

In this coordinate system, the dilaton and four-form become, with $\Delta^{2}+\delta^{2}=10$,

$$
e^{2 \phi}=e^{2 \phi_{0}}\left(\frac{w^{4}+b^{4}}{w^{4}-b^{4}}\right)^{\Delta}, C_{(4)}=-\frac{1}{4} H^{-1} d t \wedge d x \wedge d y \wedge d z .
$$

We now consider the D7-brane action in the static gauge with world-volume coordinates identified with $x_{0,1,2,3}$ and $w_{1,2,3,4}$, with transverse fluctuations parameterized by $w_{5}$ and $w_{6}$. It is convenient to define a coordinate $\rho$ such that $\sum_{i=1}^{4} d w_{i}^{2}=d \rho^{2}+\rho^{2} d \Omega_{3}^{2}$ and the radial coordinate is given by $w^{2}=\rho^{2}+w_{5}^{2}+w_{6}{ }^{2}$. The Dirac-Born-Infeld action of the D7-brane probe in this background takes the form

$$
S_{D 7}=-T_{7} \int d^{8} \xi \epsilon_{3} e^{\phi} \mathcal{G}\left(\rho, w_{5}, w_{6}\right)\left(1+g^{a b} g_{55} \partial_{a} w_{5} \partial_{b} w_{5}+g^{a b} g_{66} \partial_{a} w_{6} \partial_{b} w_{6}\right)^{1 / 2},
$$

where

$$
\mathcal{G}\left(\rho, w_{5}, w_{6}\right)=\rho^{3} \frac{\left(\left(\rho^{2}+w_{5}^{2}+w_{6}^{2}\right)^{2}+b^{4}\right)\left(\left(\rho^{2}+w_{5}^{2}+w_{6}^{2}\right)^{2}-b^{4}\right)}{\left(\rho^{2}+w_{5}^{2}+w_{6}^{2}\right)^{4}} .
$$

From these equations we derive the corresponding equation of motion. We look for classical solutions of the form $w_{6}=w_{6}(\rho), w_{5}=0$ that define the ground state. Numerically

we find solutions with the asymptotic behavior $w_{6} \sim m+c / \rho^{2}$. The identification of these constants as field theory operators requires a coordinate transformation because the scalar kinetic term is not of the usual canonical AdS form. Transforming to coordinates 11] in which the kinetic term has canonical form, we see that $m$ has dimension 1 and 
$c$ has dimension 3. These coefficients are then identified with the quark mass $m_{q}$ and condensate $\langle\bar{\psi} \psi\rangle$ respectively, in agreement with the usual AdS/CFT dictionary.

We find the physical solutions of the equation of motion by imposing the following regularity constraint: The Constable-Myers background has a naked singularity in the far IR at $\rho^{2}+w_{6}^{2}=b^{2}$ (We set $b=1$ henceforth). Thus there are two possibilities for a solution with an interpretation as an RG flow: either the D7-brane terminates at a value of $w=w_{6}(\rho=0)$ away from the naked singularity via a collapse of the $S^{3}$, or the D7-brane reaches the singularity. In the latter case we would have little control over the physics without a better understanding of string theory in such highly curved backgrounds. This applies in particular to the solution with $m=0$. For $m=0$, the solution $w_{6}=0$ is exact, which naively seems to indicate the absence of a chiral condensate $(c=0)$. However, this solution reaches the singularity, and therefore can not be trusted.

Fortunately something remarkable seems to happen. For non-zero values of $m$ we find that the regular solutions terminate before reaching the singularity. Some of these regular solutions are plotted in figure 1a. Even for a very small but non-zero mass, the regular solutions obtained numerically require a non-vanishing $c$ and terminate at $w \geq 1.35$ before reaching the singularity at $w=1$ ! This suggests that for $m=0$ there are two solutions. In addition to the unphysical solution $m=0, c=0$ which reaches the singularity, there is a regular solution $m=0, c=1.85$ which is obtained when taking the limit $m \rightarrow 0$.
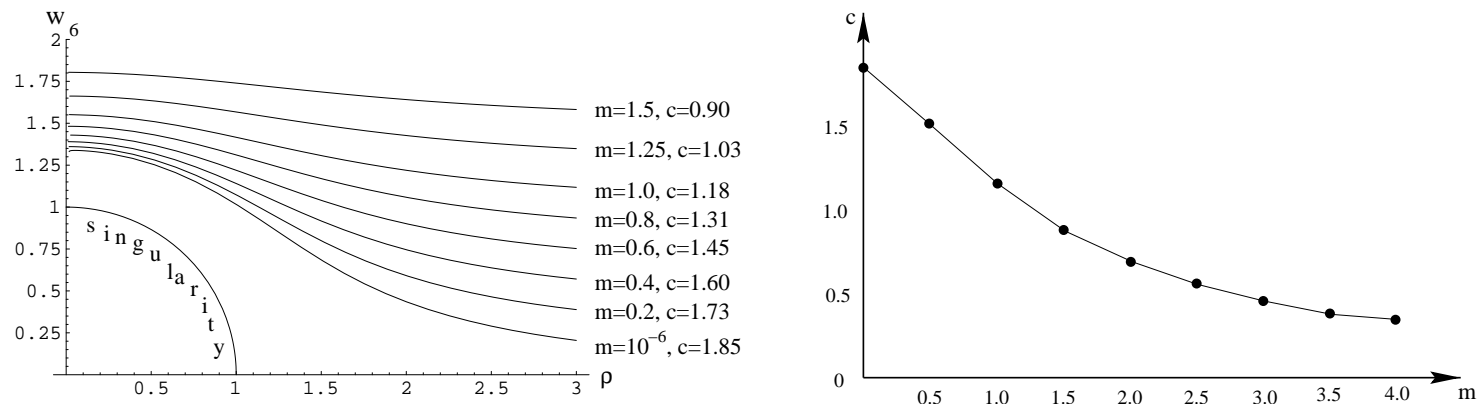

Figure 1: Regular solutions of the D7-brane probe equation of motion in the Constable-Myers background.

In figure $1 b$ we plot $c$ as a function of $m$ for the regular solutions. The numerical evidence suggests that there is a non-zero condensate in the limit $m \rightarrow 0$. This corresponds to spontaneous breaking of the $U(1)$ chiral symmetry by the quark condensate $c$.

The condensate seems to screen the probe from the naked singularity. This is reminiscent of the enhançon mechanism 22 found in $\mathcal{N}=2$ gravity duals, where the singularity of the geometry is screened from the physics of a D3-brane probe. It is possible we are seeing hints of something similar, if more complicated, here, although at this stage we can not see how to remove the singularity.

\section{$3 \quad$ Large $N$ Goldstone boson $\left(\eta^{\prime}\right)$}

Since there is chiral symmetry breaking via a condensate in the $m \rightarrow 0$ limit, we expect there to be a Goldstone boson in the meson spectrum. Such a Goldstone mode must exist as a solution to the DBI equation of motion, as the following holographic version

of the Goldstone theorem shows. Assume a D7-embedding with $w_{5}=0$ and $w_{6} \sim c / \rho^{2}$ asymptotically. A small $U(1)$ rotation $\exp (i \epsilon)$ of $w_{5}+i w_{6}$ generates a solution which is a normalizable small fluctuation about this background, with $w_{6}$ unchanged (to order $\left(\epsilon^{2}\right)$ ) 
and $w_{5}=\epsilon c / \rho^{2}$. Thus a small fluctuation with $w_{6}$ unchanged and $w_{5}=\epsilon \frac{c}{\rho^{2}} \sin (k \cdot x)$ is a normalizable solution of the linearized equations of motion provided $k^{2}=0$. Thus there must be a massless meson associated with the $w_{5}$ fluctuations. Note that if the embedding were asymptotically $w_{6} \sim m+c(m) / \rho^{2}$ for non-zero $m$, a $U(1)$ rotation of $w_{5}+i w_{6}$ would still generate another solution. However this solution is no longer a normalizable small fluctuation about the original embedding. Thus we no longer expect a massless meson, which reflects the explicit symmetry breaking by the non-zero quark mass $m$.

The existence of a Goldstone boson can be verified numerically. In the vacuum solutions discussed where $w_{6}$ has a background value, fluctuations in $w_{5}$ should contain the Goldstone mode. Therefore we solve the linearized equation of motion numerically for normalizable small fluctuations of the form $w_{5}=f(\rho) \sin (k \cdot x)$, with $x$ the four Minkowski coordinates and $k^{2}=-M^{2}$. For the regular solutions, the meson mass is a function of quark mass which is plotted in figure 3. The meson mass indeed falls to zero as the quark mass is taken to zero, providing further evidence of chiral symmetry breaking. Furthermore, at small $m$ the meson mass scales like $\sqrt{m}$. This scaling is well known in QCD, and can be derived from a chiral Lagrangian.

For comparison it is interesting to study $w_{6}$ fluctuations as well. The numerical solutions for the $w_{6}$ fluctuations are also plotted in figure 3 . The $w_{6}$ fluctuations have a mass gap, as expected since they are transverse to the vacuum manifold.
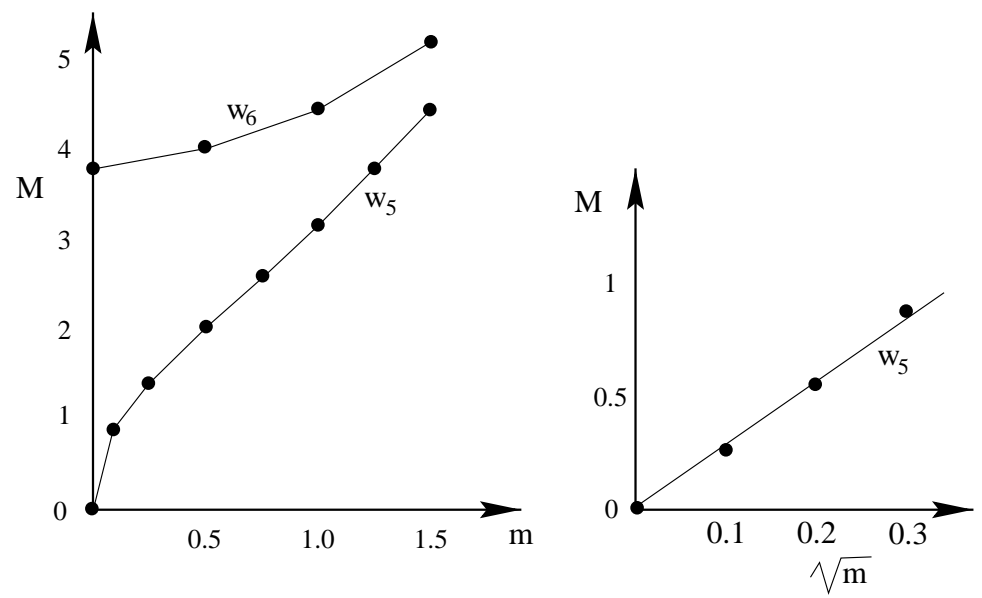

Figure 2: A plot of the $w_{5}$ and $w_{6}$ meson mass vs quark mass $m$ associated with the fluctuations about the regular solutions of the equation of motion for the Constable-Myers flow. The Goldstone mass is also plotted vs $\sqrt{m}$ with a linear fit.

Note that the $U(1)$ chiral symmetry is non-anomalous only in the limit $N \rightarrow \infty$, which is also the limit where classical supergravity and Dirac-Born-Infeld theory are reliable. The Goldstone boson discussed above is analogous to the $\eta^{\prime}$ in QCD, which becomes a Goldstone boson in the large $N$ limit [16]. We expect that finite $N$ stringy effects will give the $\eta^{\prime}$ a non-zero mass. A presently unsolved challenge is to obtain a background corresponding to spontaneously broken $U\left(N_{f}\right) \times U\left(N_{f}\right)$ chiral symmetry for $N_{f}>1$. Embedding $N_{f}$ D7-branes in the Constable-Myers background gives a diagonal $U\left(N_{f}\right)$ and an axial $U(1)$. There is no larger chiral symmetry because of couplings $\tilde{\psi} X \psi$ which involve adjoint scalars $X$. Note that the axial $U(1)$ symmetry also acts on $X$.

It is nevertheless interesting to make a rough comparison between our $\eta^{\prime}$ and the QCD pions: In a large $N$ QCD model with more than one degenerate quark flavor, there would be $N_{f}^{2}$ degenerate Goldstone bosons including the usual pions as well as $\eta^{\prime}$. In standard 
QCD the bare up or down quark mass is roughly $0.01 \Lambda$ and the pion mass of order $0.5 \Lambda$ (it is of course hard to know precisely what value one should pick for the strong-coupling scale $\Lambda$ ). If we assume that $\Lambda \simeq b=1$ then for this quark mass of $0.01 \Lambda$ we find $m_{\pi} \simeq 0.25 \Lambda$. The gravity dual is correctly predicting the pion mass at the level of a factor of two. Of course we cannot expect a perfect match, given the large $N$ limit as well as the additional degrees of freedom which are present in the deformed $\mathcal{N}=4$ theory.

In conclusion, we have demonstrated that holography captures one of the most important qualitative features of a confining QCD-like theory, namely chiral symmetry breaking by a quark condensate. So far we have resorted to numerics for solving the relevant equations of motion. A natural next step is to understand the main features of the solutions analytically. Moreover it would be interesting to see if chiral symmetry breaking may

be obtained in even more realistic backgrounds. This would open up the possibility of studying the light mesonic sector of QCD using the new techniques presented here.

Acknowledgement We are very grateful to R. Brower, N. Constable, A. Hanany, C. Núñez, M. Petrini and N. Prezas for enlightening discussions. The research of J.E., Z.G. and I.K. is supported by DFG within the 'Emmy Noether' programme. J.B. is grateful for support through the A. von Humboldt Foundation and N.E. for the support of a PPARC Advanced Research Fellowship.

\section{References}

[1] J.M. Maldacena, Adv. Theor. Math. Phys. 2 (1998) 231 arXiv:hep-th/9711200.

[2] E. Witten, Adv. Theor. Math. Phys. 2 (1998) 505 arXiv:hep-th/9803131.

[3] S. S. Gubser, arXiv:hep-th/9902155.

[4] J. Babington, D. E. Crooks and N. Evans, JHEP 0302 (2003) 024 arXiv:hep-th/0207076; J. Babington, D. E. Crooks and N. Evans, Phys. Rev. D 67 (2003) 066007 arXiv:hep-th/0210068; N. Evans, "QCD-like Gauge Dynamics From Gravity Duals," same proceedings.

[5] N. R. Constable and R. C. Myers, JHEP 9911 (1999) 020 arXiv:hep-th/9905081;

[6] C. Csaki, H. Ooguri, Y. Oz and J. Terning, JHEP 9901, 017 (1999) arXiv:hep-th/9806021; R. C. Brower, S. D. Mathur and C. I. Tan, Nucl. Phys. B 587 (2000) 249 arXiv:hep-th/0003115.

[7] M. Teper, arXiv:hep-ph/0203203; C. J. Morningstar and M. J. Peardon, Phys. Rev. D 60, 034509 (1999) |arXiv:hep-lat/9901004; D. E. Crooks and N. Evans, arXiv:hep-th/0302098.

[8] A. Karch and L. Randall, JHEP 0106 (2001) 063 arXiv:hep-th/0105132.

[9] O. DeWolfe, D. Z. Freedman and H. Ooguri, Phys. Rev. D 66 (2002) 025009 arXiv:hep-th/0111135.

[10] N. R. Constable, J. Erdmenger, Z. Guralnik and I. Kirsch, arXiv:hep-th/0211222.

[11] A. Karch and E. Katz, JHEP 0206 (2002) 043 arXiv:hep-th/0205236;

[12] M. Kruczenski, D. Mateos, R. C. Myers and D. J. Winters, arXiv:hep-th/0304032.

[13] T. Sakai and J. Sonnenschein, JHEP 0309 (2003) 047 arXiv:hep-th/0305049. 
[14] J. Babington, J. Erdmenger, N. Evans, Z. Guralnik and I. Kirsch, arXiv:hep-th/0306018.

[15] S. S. Gubser, Adv. Theor. Math. Phys. 4 (2002) 679 arXiv:hep-th/0002160.

[16] E. Witten, Nucl. Phys. B 156 (1979) 269.

[17] X. J. Wang and S. Hu, JHEP 0309 (2003) 017 arXiv:hep-th/0307218.

[18] P. Ouyang, arXiv:hep-th/0311084.

[19] C. Nunez, A. Paredes and A. V. Ramallo, arXiv:hep-th/0311201.

[20] M. Kruczenski, D. Mateos, R. C. Myers and D. J. Winters, arXiv:hep-th/0311270.

[21] S. Hong, S. Yoon and M. J. Strassler, arXiv:hep-th/0312071.

[22] C. V. Johnson, A. W. Peet and J. Polchinski, Phys. Rev. D 61 (2000) 086001 arXiv:hep-th/9911161. 